\title{
Effects of Learning by Video Modeling on Gymnastic Performances among Tunisian Students in the Second Year of Secondary Level
}

\author{
Majdi Bouazizi ${ }^{1}$, Fairouz Azaiez ${ }^{2}$ And Driss Boudhiba ${ }^{2}$ \\ ${ }^{1}$ Faculty of Sciences of Bizerte, Tunisia \\ ${ }^{2}$ High Institute of Sport and Physical Education of Sfax, Tunisia
}

\begin{abstract}
The purpose of this work was to analyze the effects of learning through video modeling combined of sessions of physical education on gymnastic performance among Tunisian students in the second year of secondary level. Our study was conducted on a sample of 103 subjects (16,95 \pm 0,9 years) divided into two independent groups (experimental and control). The experimental group was subjected to a cycle of video modeling with explanations and verbal representations of gymnastic skills combined with sessions of physical education gymnastics, while the second group was practiced gymnastics with the usual conditions in the physical education sessions. Measures of performances were taken before and after the experiment. These results demonstrate the effectiveness of learning by video modeling procedures for the acquisition and improvement of gymnastic skills for students. In addition, the progression of gymnastic performance of girls is more important than boys after receiving the learning by video modeling.
\end{abstract}

Keywords: Learning; Video modeling; Performance; Gymnastics

\section{Introduction}

Bandura $(1969,1977)$, the foremost proponent of modeling strategies, has identified four components that mediate observational learning: attention to modeled events, retention of what is observed, ability to replicate modeled behaviors, and motivation to reproduce those behaviors. Several characteristics of the model have been shown to affect the mediation of these four components, one being the status of the model (McCullagh, 1986). Other characteristics are the use of similar models, multiple models, and coping models (Dowrick \& Jesdale, 1991).

Thanks to the advanced technology, video viewing of a model or on its own performance has already been demonstrated in several studies (Horn et al., 2002; Merian and Baumberger, 2007; Baudry et al., 2006) as beneficial for learning, either immediately or in the long term (Guadagnoli, 2002).

Behavioral procedures have proven to be effective in improving athletic performance across a diverse array of sports, including ballet (Fitterling \& Ayllon, 1983), basketball (Harle \& Vickers, 2001), tennis (Rikli \& Smith, 1980), swimming (McKenzie \& Rushall, 1974), football (Smith \& Ward, 2006), and gymnastics (Wolko, Hrycaiko, \& Martin, 1993). Two behavioral procedures used for improving skill execution are video feedback and video modeling by experts (Hazen, Johnstone, Martin, \& Srikameswaran, 1990). Video feedback involves showing an athlete a video clip of his or her own performance of a particular skill (Hazen et al.), and video modeling involves presenting the athlete with a video clip of an expert performing the skill (Boschker \& Bakker, 2002; SooHoo, Takemoto, \& McCullagh, 2004; Winfrey \& Weeks, 1993; Zetou, Tzetzis, Vernadakis, \& Kioumourtzoglou, 2002).

The combined use of video modeling and feedback holds promise for improving the execution of complex athletic skills such as gymnastics routines that require multiple precise body movements and positions. Only two studies have examined the effectiveness of the combination of video feedback and video modeling by experts. Harle and Vickers (2001) helped basketball players improve eye gaze at the hoop to increase free-throw accuracy, whereas Rikli and Smith (1980) helped adult tennis players improve their serves. Because one study was conducted on a simple skill involving sustained eye gaze and the other study involved a group design that showed minimal effects, the effects of video feedback and video modeling by experts on complex athletic performance are not well established. The purpose of the present study was to examine the effectiveness of combining video modeling by experts with video feedback on the development of three complex gymnastics skills.

\section{Participants}

\section{Materials and methods}

This study was conducted on one hundred three Tunisian pupils (16,95 $\pm 0,9$ years); 49 males and 54 females studying in second year of secondary level. They were divided into two groups of fifty-two and fifty- 
one; the first group has been submitted to the video modeling of gymnastic skills and the last one served as control.

\section{Materials}

The materials included a digital video camera to record participants' performance in the two assessment sessions, a laptop and a video projector to view the clips demonstrating the gymnastic skills by the model.

The experiment was conducted in a gymnastics area of school with the usual conditions of gymnastic sessions of practice.

\section{Procedure}

The experimental group received learning by video of gymnastic skills combined of sessions of physical education in contrast to the control group who practiced gymnastics with habitual conditions according to the distribution of the institution.

Predictive assessment (taking performance) took place before the start of the gymnastic cycle, even for a second evaluation was conducted at the end of the cycle. During the viewing it was clearly explained the different success criteria for each skill of the gymnastic sequence: handstand, forward roll, backward roll, wheel, face plate. The teacher insists on the important technical points and the criteria for success of motor skills in video sequences of gymnastic.

During the evaluation of the beginning of the cycle, the students had to perform a series of five gymnastic elements which constitute the basic requirements usually taught at this level of schooling. Students were videotaped and the recording is presented to physical education teachers for evaluation and awarding marks. An end score is assigned to each student by averaging all the marks awarded by the evaluators.

Each skill is accompanied by success criteria to meet determining its level of performance. The achievement of each gymnastic skills (the stretched handstand, the roll forward, backward roll, the wheel and the face plate) is recorded on 4 points. The performance has been calculated by the average score of gymnasts attributed by evaluator teachers.

\section{Statistical analyses}

The Analysis of variance (ANOVA) of results was made following a randomly complete block design (Snedecor and Cochran, 1957) by the GLM procedure of the SAS statistical package (SAS Institute, 1989). Homogeneity of variance was verified by the standard Bartlett test (Anderson and McLean, 1974). All statistics are considered significant for a probability level less than $0.05(\mathrm{p}<0.05)$.

\section{Results}

Figures 1 and 2 show that the progression of gymnastic performances of group benefiting from learning by video modeling is significantly better than the control group.

In fact at the beginning of gymnastics cycle the performance of both experimental and control groups are approximately equal, while the performance of the end of the cycle are significantly better among students who benefited by submission of video clips of modeling gymnastic skills $(p \leq 0.001$; Fig 1$)$. Therefore the progression performance of the experimental group was significantly higher than the control group (9.61 vs 8.38).

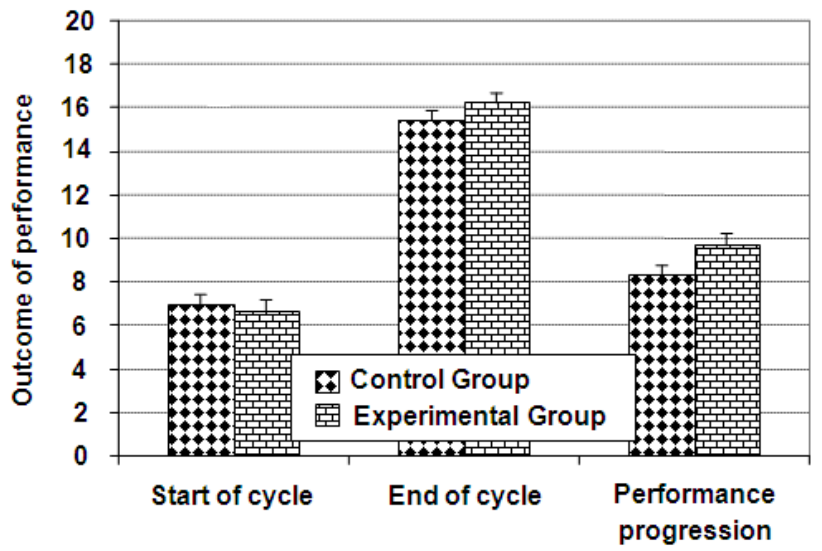

Figure 1. The evolution of gymnastic performances for the two groups before and after submission to learning by video modeling of motor skills 
The results of this study indicate that exposure to the video modeling improved skill performance more than habitual practice. The analysis of variance indicate that gymnastic progressions of girls are superior to progressions of boys after the submission cycle modeling video gymnastic skills $(10,43$ vs 8,93$)$.

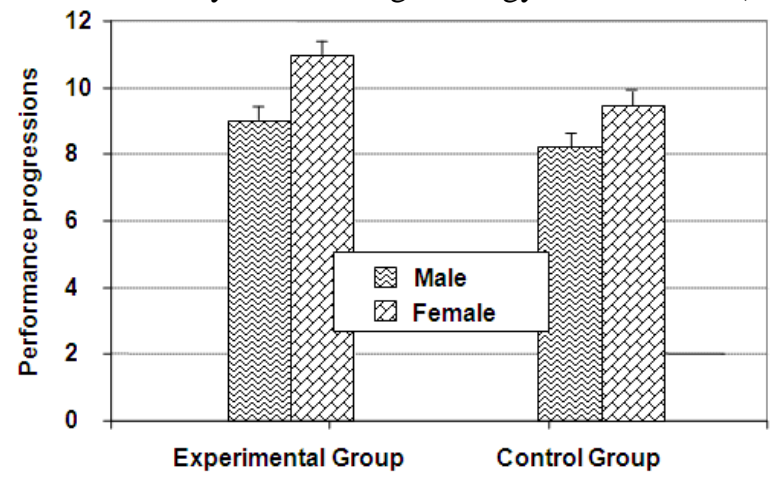

Figure 2. The progressions of gymnastic performances of males and females after the submission to learning by video modeling

\section{Discussion}

We noticed that learning with video modeling of gymnastic skills has a positive effect on improving student performance. These results are confirmed by the research of Baudry et al. (2006) who argue that the video modeling helps the gymnast to develop a cognitive representation of movement. Viewing sequences, the subject will analyze the steps necessary to achieve the motor performance. It will treat and build, somehow, a procedure to achieve the goal. This will allow him to have a better understanding of the driving task and monitor the progress. After viewing the images, students are better able to understand the movement and particularly in terms of the sequence. In the example of a complex movement, which would require the sequence of several actions, they would be more likely to put the steps in order.

These results support those found by the search for Li-Wei, et al. (1992); this is one of the few field studies in which were analyzed the effects of the application of a mental training program using video observation model for improving sports skills in children. There was significant improvement in performance of the attack forehand in table tennis with children 7 to 10 years.

In view of the newly developed, the video viewing has high efficiency for the understanding of the engine kinematic movements. The latter being also known to help the player to build an image of the movement executed (Bertsch and The Scanff, 1995).

Previous studies concluded that video modeling and video feedback could improve athletic skills execution (Harle \& Vickers, 2001; Hazen et al., 1990). In 1990, Hazen et al. implemented an intervention package that included modeling, role-playing, symbolic modeling (expert video modeling), instructions, self video feedback, and verbal feedback.

Research of Baudry et al. (2006) argue that the video model helps the gymnast to develop a cognitive representation of movement. Viewing sequences, the subject will analyze the steps necessary to achieve the performance. It will treat and build, somehow, a procedure to achieve the goal. This will allow him to have a better understanding of the driving task and monitor the progress. After viewing the images, students are better able to understand the movement and particularly in terms of the sequence. In the example of a complex movement, which would require the sequence of several actions, they would be more likely to put the steps in the order.

The findings add to the scant literature supporting the effectiveness and usefulness of the procedure (e.g., Harle \& Vickers, 2001; Hazen et al., 1990; Rikli \& Smith, 1980) and suggest its potential to enhance the effects of coaching across a variety of sports.

We believe that video modeling and video feedback for gymnasts has the most potential to be effective for increasing the execution of a skill that has already been learned at a basic performance level, as was found by Rikli and Smith (1980).

\section{References}

[1]. Bandura A. 1969. Principles of behavior modification. Englewood Cliffs, NJ: Prentice-Hall.

[2]. Bandura A. 1977. Social learning theory. New York: Holt, Rinehart, \& Winston.

[3]. Baudry L., Leroy D., Chollet D. 2006. The effect of combined self- and expertmodelling on the performance of the double leg circle on the pommel horse. Journal of Sports Sciences, 24:1055-1063.

[4]. Bellini S., Akullian J. 2007. A metaanalysis of video modeling and video self-modeling interventions for children and adolescents with autism spectrum disorders. Exceptional Children, 73:264-287.

[5]. Boschker M. C. J., Bakker F. C. 2002. Inexperienced sport climbers might perceive and utilize new opportunities for action by merely observing a model. Perceptual and Motor Skills, 95:3-9. 
[6]. Dowrick P. W., Jesdale D. C. 1991. Modeling. In P. W. Dowrick (Ed.), Practical guide to using video in the behavioral sciences. New York: Wiley. 64-76.

[7]. Fitterling J. M., Ayllon T. 1983. Behavioral coaching in classical ballet: Enhancing skill development. Behavior Modification, 7:345-368.

[8]. Guadagnoli M.A., Holcomb W., Davis M. 2002. The efficacy of video feedback for learning the golf swing. Journal of Sports Sciences, 20:615-622.

[9]. Harle S. K., Vickers J. N. 2001. Training quick eye improves accuracy in the basketball free throw. The Sport Psychologist, 15:289-305.

[10]. Hazen A., Johnstone C., Martin G. L., Srikameswaran S. 1990. A videotaping feedback package for improving skills of youth competitive swimmers. The Sport Psychologist, 4:213-227.

[11]. Horn R. R., Williams A. M., Scott M. A. 2002. Learning from demonstrations: the role of visual search during observational learning from video and point-light models. Journal of Sports Sciences, 20:253-69.

[12]. Merian T., Baumberger B. 2007. Le feedback vidéo en éducation physique scolaire. Staps, 76:107-120.

[13]. McCullagh P. 1986. Model status as a determinant of observational learning and performance. Journal of Sport Psychology, 8:319-331.

[14]. McKenzie T. L., Rushall B. 1974. Effects of selfrecording on attendance and performance in a competitive swimming training environment. Journal of Applied Behavior Analysis, 7:199-206.

[15]. Smith S. L., Ward P. 2006. Behavioral interventions to improve performance in collegiate football. Journal of Applied Behavior Analysis, 39:385-391.

[16]. Rikli R., Smith G. 1980. Videotape feedback effects on tennis serving form.

[17]. Ripoll H., La Rue J. 2004. Manuel de psychologie du sport. Editions Revue EPS-11, tome 1:85-112.

[18]. SooHoo S., Takemoto K. Y., McCullagh P. 2004. A comparison of modeling and imagery on the performance of a motor skill. Journal of Sport Behavior, 27:349-366.

[19]. Winfrey M. L., Weeks D. S. 1993. Effects of self modeling on self-efficacy and balance beam performance. Perceptual and Motor Skills, 77:907-913.

[20]. Wolko K. L., Hrycaiko D. W., Martin G. L. 1993. A comparison of two self-management packages to standard coaching for improving practice performance of gymnasts. Behavior Modification, 17:209-223.

[21]. Zetou E., Tzetzis G., Vernadakis N., Kioumourtzoglou E. 2002. Modeling in learning two volleyball skills. Perceptual and Motor Skills, 94:1131-1142. 